\title{
IMAGES OF HOMOGENEOUS VECTOR BUNDLES AND VARIETIES OF COMPLEXES
}

\author{
BY GEORGE R. KEMPF ${ }^{1}$
}

Communicated by Robert Fossum, April 21, 1975

Let $G$ be a connected algebraic group with a given representation on a vector space $V$. Let $W$ be a subspace of $V$. I propose to study the union of all the translates of $W$ by $G, G \cdot W$.

Let $P$ be a subgroup of $G$ that stabilizes $W$. Let $X \rightarrow G / P$ be the homogeneous vector bundle over $G / P$, associated to the representation of $P$ on $W$. Explicitly

$$
X=\left\{(g, w) \in G \times W \text { modulo }(g, w) \sim\left(g p^{-1}, p w\right) \text { for } p \in P\right\}
$$

The representation $G \times V \rightarrow V$ induces a morphism $f: X \rightarrow V$. The image of $f$ is $G \cdot W$.

TheOREM. Assume $G / P$ is complete. Then $G \cdot W$ is a closed subvariety of $V$. Furthermore, if the characteristic of the ground field is zero, and if the actions of $G$ on $V$ and of $P$ on $W$ are completely reducible, then $G \cdot W$ is a normal CohenMacaulay variety, and if $f$ is birational, then $G \cdot W$ has rational singularities.

The proof of this theorem uses the Borel-Weil-Bott theorem on the cohomology of homogeneous vector bundles [1] together with some facts surrounding the theory of rational resolutions [5].

The application that $I$ have in mind for this theorem is the study of the singularities of the varieties of complexes introduced by Buchsbaum and Eisenbud [2].

I will first state what these varieties are. Let $K^{0}, \ldots, K^{n}$ be a sequence of vector spaces. Let $V$ be the direct sum of $\operatorname{Hom}\left(K^{0}, K^{1}\right), \ldots, \operatorname{Hom}\left(K^{n-1}, K^{n}\right)$. A point $a$ in $V$ is denoted $\left(a_{1}, \ldots, a_{n}\right)$, where $a_{i} \in \operatorname{Hom}\left(K^{i-1}, K^{i}\right)$. A point $a$ in $V$ represents a complex if $a_{i+1} \circ a_{i}=0$ for $0<i<n$. The rank of $a$ is the sequence of integers, (rank $a_{1}, \ldots$, rank $a_{n}$ ), where rank $b$ is the dimension of the image of the homomorphism $b$. If $\left(m_{1}, \ldots, m_{n}\right)$ is the rank of a complex, then $m_{1} \leqslant \operatorname{dim} K^{0}, m_{n} \leqslant \operatorname{dim} K^{n}$, and $m_{i}+m_{i+1} \leqslant \operatorname{dim} K^{i}$ for $0<i<n$. Conversely, any such sequence is the rank of a complex. Let $M$ be the set of such sequences.

If $m \in M$, define the variety of Buchsbaum and Eisenbud, $\mathrm{B}-\mathrm{E}(m)$, to be the variety of complexes $a$, such that rank $a_{i} \leqslant m_{i}$ for $1 \leqslant i \leqslant n$.

AMS (MOS) subject classifications (1970). Primary 14M05, $14 \mathrm{M} 15$.

1 Partly supported by the Sloan Foundation. 
THEOREM. In characteristic zero, a Buchsbaum-Eisenbud variety of complexes is a normal Cohen-Macaulay variety and has rational singularities.

Of course, this last theorem should hold in any characteristic. The ideal of functions vanishing on $\mathrm{B}-\mathrm{E}(\mathrm{m})$ should be generated by the quadric relations, corresponding to the complex condition, and the determinants, corresponding to the rank conditions. One should be able to prove that these functions generate a prime ideal by the inductive method used by Eagon and Hochster [3] where they settle the case when $n=1$ (see also [4]).

In the rest of this note, I will sketch how the first theorem impiies the second. I plan to publish the first theorem separately.

Let $G=\mathrm{GL}\left(K^{1}\right) \times \ldots \times \mathrm{GL}\left(K^{n}\right)$. Consider a sequence $L=\left(L^{i}, \ldots, L^{n}\right)$, where $L^{i}$ is a subspace of dimension $m_{i}$ of $K^{i}$. Let $W(L)$ be the subspace of $V$ consisting of $a$ 's such that the image of $a_{i} \subset L^{i} \subset$ the kernel of $a_{i+1}$ whenever either statement makes sense. Let $J$ be a fixed sequence of subspaces as above. Let $W=W(J)$. Let $P$ be the product of the stabilizer of $J^{i}$ in $\operatorname{GL}\left(K^{i}\right)$ for $1 \leqslant$ $i \leqslant n$. $P$ stabilizes $W$. The homogeneous vector bundle $X$ is the totality of all pairs $L$ and $a$ such that $a \in W(L)$.

$G / P$ is the space of $L$ 's, which is a product of Grassmannian. Hence, $G / P$ is complete. The mapping $f$ sends $(L, a)$ onto $a$. The image is clearly all of $\mathrm{B}-\mathrm{E}(m)$. If a point $a \in \mathrm{B}-\mathrm{E}(m)$ satisfies the rank conditions exactly, there is a unique pair $(L, a)$ in $X$. In fact, $L^{j}$ must be the image of $a_{j}$. The morphism $f$ is birational because it is an isomorphism near any such "general" point of $\mathrm{B}-\mathrm{E}(m)$.

It remains to verify to complete reducibility assumptions. Assume the characteristic is zero. Then, as $G$ is a reductive group, any of its representations are completely reducible. As for $P$ acting on $W$, note that $a \in W$ is determined by the induced homomorphisms

$$
K^{0} \rightarrow J^{1}, K^{1 / J^{1}} \rightarrow J^{2}, \ldots, K^{n-1} / J^{n-1} \rightarrow J^{n} .
$$

Thus, the action of $P$ on $W$ is equivalent to the action of its quotient $\operatorname{GL}\left(J^{1}\right) \times$ $\mathrm{GL}\left(K^{1} / J^{1}\right) \times \ldots \times \mathrm{GL}\left(J^{n}\right)$, which is again reductive.

Therefore, we have not only found a pleasant resolution of the singularities of B-E $(m)$, but we conclude that the first theorem implies the second.

\section{REFERENCES} 19,681 .

1. R. Bott, Homogeneous vector bundles, Ann. of Math. (2) 66 (1957), 203-248. MR

2. D. Buchsbaum and D. Eisenbud, Unpublished research.

3. M. Hochster and J. A. Eagon, Cohen-Macaulay rings, invariant theory, and the generic perfection of determinatal loci, Amer.J. Math. 93 (1971), 1020-1058. MR 46 \#1787.

4. G. Kempf, Vanishing theorems for flag manifolds (to appear).

5. G. Kempf, F. Knudsen, D. Mumford and B. St. Donat, Toroidal embeddings. I, Lecture Notes in Math., vol. 339, Springer-Verlag, New York, 1973.

DEPARTMENT OF MATHEMATICS, JOHNS HOPKINS UNIVERSITY, BALTIMORE, MARYLAND 21218 